\title{
Impact of NBTI Recovery, Measurement System and Testing Time on NBTI Lifetime Estimation
}

\author{
Vincent King Soon $\mathrm{WONG}^{1}$, Hong Seng $\mathrm{NG}^{1}$ and Poh Ching SIM ${ }^{2}$ \\ ${ }^{1}$ Reliability, Quality, X-FAB Sarawak Sdn Bhd, Kuching, Sarawak, Malaysia \\ ${ }^{2}$ Process Developments, X-FAB Sarawak Sdn Bhd, Kuching, Sarawak, Malaysia
}

\begin{abstract}
Negative Bias Temperature Instability (NBTI) measurements had always been a challenge for reliability lifetime estimations. Currently various methods had been developed to suppress this characteristic but being able to completely remove it had been to no avail. This paper will first demonstrate the degree of impact of recovery on NBTI measurements; and then moving on to the influence of measurement system as well as testing time on NBTI lifetime estimation.
\end{abstract}

\section{Keywords-NBTI; recovery; reliability; device lifetime}

\section{INTRODUCTION}

The semiconductor world had significantly evolved over the recent years as device scaling continues to excel in the nanometer range. One of the catalysts to such progress is through the use of ultra-thin gate oxides. However, gate oxide shrinkage infers a vital reliability concern which is the transistor NBTI performances. Typically thinner gate oxides will induce much substantial NBTI degradation [1]. Hence it is absolutely important to effectively and accurately measure the NBTI performance of a device to better characterize its reliability.

The conventional methods of NBTI evaluation is done by applying stress voltage to the gate of a transistor and then measure the threshold voltage $\left(\mathrm{V}_{\mathrm{t}}\right)$ changes over time. However due to fast recovery mechanism, the NBTI degradations recover almost instantaneously to a certain extent once the gate stress was lifted [2]; thereby complicating the characterization of the device actual NBTI behavior.

Therefore with the aim of acquiring accurate lifetime estimations, reliable NBTI test systems and methods are crucial factors that must be improvised. Among the currently applied solutions are recovery effect correction [3], ultra-fast switching measurements [4], improved-predictability of NBTI degradation through improved circuit simulation [5], advanced On-The-Fly Method [6] and etcetera. Nonetheless, even with the employment of slow measurement systems, NBTI lifetime estimations can still be drastically improved as shown in the investigation results in the subsequent sections.

\section{NBTI RECOVERY}

Classically NBTI testing is done by stressing the transistor gate with certain voltage for a specified period of time and upon completion, the stress voltage will be lifted while at the same time initiating the measurement sweep voltage to obtain the $\mathrm{V}_{\mathrm{t}}$. The process is repeated for the consequent pre-defined stressing periods. Thus generally the entire process involves two main stages which is the stressing stage and the measurement stage. NBTI recovery occurs during the transition in between the two stages [7]. As a result the longer the relaxation period or the buffer period in between the stressing stages, the larger the magnitude of recovery will happen on the NBTI degradation. The combination of high delay and low stress times leads to the bending of Vt-shift versus stress time degradation curve; resulting in overestimation of lifetime in the case of fast degradation or underestimation of lifetime in the case of slow degradation [3].

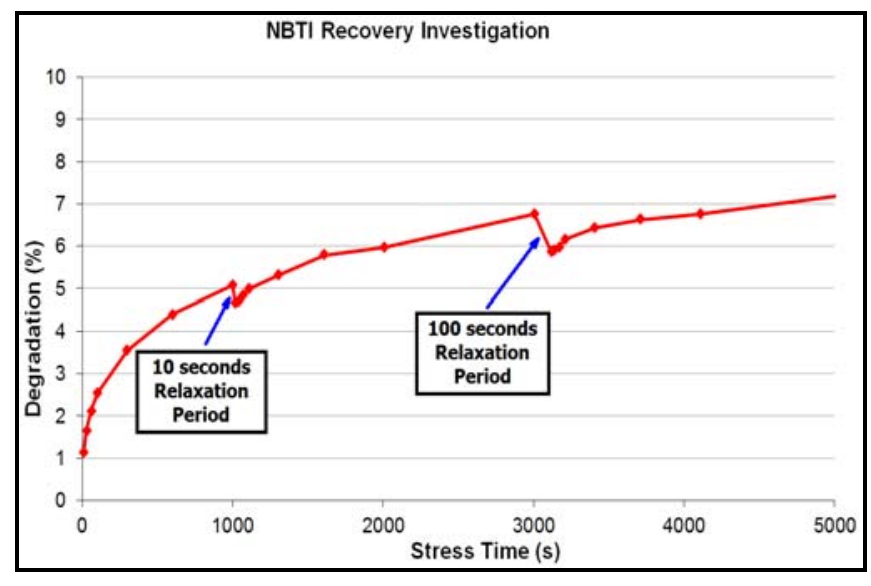

FIGURE I. IMPACT FO RELAXATION PERIOD ON NBTI DEGRADATION

Figure 1 shows the impact of different relaxation periods on NBTI degradation measurements. By varying the relaxation period after each stressing stages, the extent of recovery can be demonstrated. From the results it can be observed that the longer the relaxation period, the greater the drop in measured NBTI degradation for the subsequent point due to recovery mechanism. Hence the overall degradation will be smaller than the actual performance; which will induce overestimation of the device lifetime and consequently projects wrongful perception that the device is robust.

\section{NBTI MEASUREMENT SYSTEM}

Measurement systems applied in NBTI investigations are vital aspects that determine the accuracy of the lifetime outcomes. Fast measurement systems are very much desirable in order to better characterize NBTI behavior. Based on the Jedec Standards [8], minimizing the interim off-stress time $\left(T_{\text {off-stress }}\right)$ measurement between the termination of the NBTI 
stress and the beginning of device parameter measurements is recommended to subdue the effect of NBTI recovery. Through fast switching in between the stressing and measuring stages, the effect of recovery can be suppressed and minimized; thus resulting in better quantification of the NBTI degradations which is much nearer to the actual device performance.

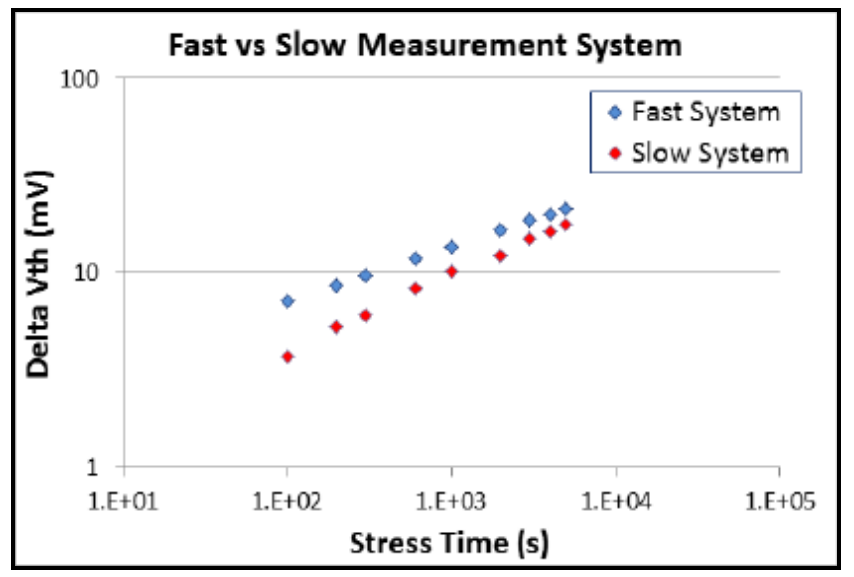

FIGURE II. IMPACT OF MEASUREMENT SYSTEM

Figure 2 shows the differences in between fast and slow measurement systems. Slow measurement system simply implies more NBTI recovery effect occurrence due to slow switching in between the stress and measurement stages. Hence the measured NBTI degradation will be contrastingly smaller than the fast system and tend to deviate more than the actual performance of the device. Therefore it is extremely important to minimize or if possible totally curb the effect of NBTI recovery so as to obtain reliable and accurate device characteristics.

\section{NBTI TESTING TIME}

The absolute ideal case to obtain NBTI lifetime is to test the device right to failure. But it is rather unfeasible as these testing consume significant time, resources and effort. Hence accelerated testing is therefore employed through higher stress application for a shorter length of time. However this shortened testing period is an important criterion that has a huge impact in lifetime extrapolation as shown in Figure 3.

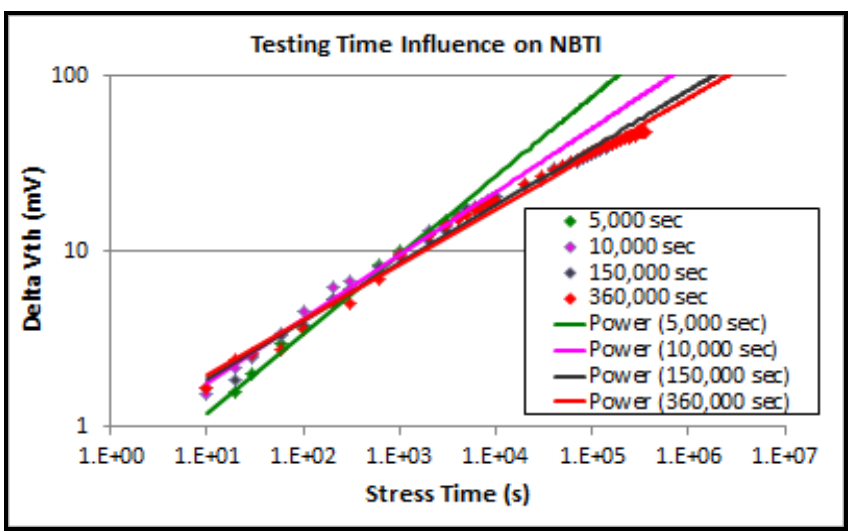

FIGURE III. INFLUENCE OF TESTING TIME ON NBTI LIFETIME EXTRAPOLATION
It can be observed that even though similar device was tested under identical stress conditions with only variations in testing periods, the results yield different degradation slopes which projects very different lifetime values. Devices that are tested longer tend to extrapolate larger lifetimes compared to shorter test periods. Among the reasons for such occurrences are measurement noises as well as NBTI recovery mechanisms which are much more significant in the initial stages. From Figure 3 it can be witnessed that devices that undergo longer test periods have degradation changes experiencing saturation after the $1.0 \mathrm{E}+05$ seconds region. This implies further testing will have minor deviations in terms of slope and lifetime extrapolations. For that reason testing beyond this region is considered sufficient to represent the NBTI characteristics of the particular device.

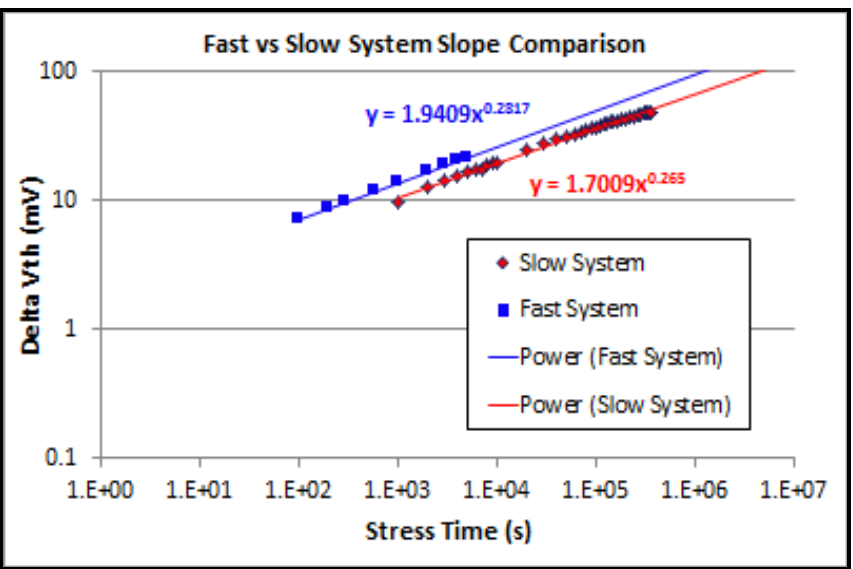

FIGURE IV. SLOPE COMPARISON IN BETWEEN FAST AND SLOW MEASUREMENT SYSTEMS

Figure 4 shows a comparison in between the slopes of a fast and slow measurement system. By extending the testing period of the slow system, the NBTI degradation slope of a device can be better approximated up to the clarification of a fast system. However as shown in this instance, in order to reach the slope or resolution of a 5,000 seconds measurement done by fast system, it takes about 360,000 seconds on a slow system. Therefore it is rather unfeasible unless testing capacity is not an issue.

\section{CONCLUSION}

As a conclusion, the implications of NBTI recovery had been demonstrated and through the use of fast measurement systems, better quantification of device NBTI characteristics can approximated at higher degree of accuracy. Nonetheless, slow measurement systems can also produce well results even though consumes much more effort and resource.

\section{REFERENCES}

[1] Yang, J., Chen, T., Tan, S., \& Chan, L., "A novel empirical model for NBTI recovery with the modulated measurement time frame." 13thInternational Symposium on the Physical and Failure Analysis of Integrated Circuits, pp. 33-36, DOI: 10.1109/IPFA.2006.250991. Singapore: IEEE Conference Publications.

[2] He, Y., "Effect of variable body bias technique on pMOSFET NBTI recovery." Electronics Letters, Volume: 45, Issue: 18, pp. 956 - 957, IET. 
[3] Reisinger, H., Vollertsen, R., Wagner, P.-J., Huttner, T., Martin, A., Aresu, S., Gustin, W., Grasser, T., Schlunder, C., "A study of NBTI and short-term threshold hysteresis of thin nitrided and thick non-nitrided oxides.” Device and Materials Reliability, IEEE Transactions on, Volume: 9, Issue: 2, pp. 106 - 114, IEEE.

[4] Du, G., Ang, D., Hu, Y., Wang, S., \& Ng, C., "Physical framework for NBTI: Insight from ultra-fast switching measurement of NBTI recovery. “ IEEE International Reliability Physics Symposium, 2008. IRPS 2008. pp. 735 - 736, Phoenix, AZ: IEEE.

[5] Benard, C., Ogier, J., \& Goguenheim, D., "Advanced on-the-fly method with correction of initial values to characterize negative bias temperature instability reliability." IEEE International Integrated Reliability Workshop Final Report, 2008. IRW 2008, pp. 12 - 15, S. Lake Tahoe, CA: IEEE.

[6] Kufluoglu, H., Reddy, V., Marshall, A., Krick, J., Ragheb, T., Cirba, C., Krishnan, A., Chancellor, C., "An extensive and improved circuit simulation methodology for NBTI recovery." IEEE International Reliability Physics Symposium (IRPS), 2010, pp. 670 - 675, Anaheim, CA: IEEE.

[7] Chiu, J.-P., Liu, Y.-H., Hsieh, H.-D., Li, C.-W., Chen, M.-C., \& Wang, T., "Statistical characterization and modeling of the temporal evolutions of $\triangle \mathrm{Vt}$ distribution in NBTI recovery in nanometer MOSFETs." IEEE Transactions on Electron Devices, pp. 978 - 984, DOI: 10.1109/TED.2013.2240390, IEEE.

[8] Jedec Standard JESD90 "A Procedure for Measuring P-Channel MOSFET Negative Bias Temperature Instabilities" 